\title{
LA INFLUENCIA DE LAS HERRAMIENTAS DIGITALES EN LA INTELIGENCIA EMOCIONAL DE LOS NIÑOS DE INICIAL
}

\author{
THE INFLUENCE OF DIGITAL TOOLS ON THE EMOTIONAL INTELLIGENCE OF PRESCHOOL CHILDREN \\ Rosario Aguirre-Bardales ${ }^{1}$, Gloria Flores-Pérez ${ }^{2}$ \\ https://doi.org/10.52109/cyp2022326 \\ ${ }^{1}$ Facultad de Humanidades y Ciencias Sociales de la Universidad Ciencias y Humanidades, \\ rosaguirreb@uch.pe \\ ${ }^{2}$ Facultad de Humanidades y Ciencias Sociales de la Universidad Ciencias y Humanidades, glofloresp@uch.pe
}

REGISTROS

Recibido el 16/10/2021

Aceptado el 27/01/2022

Publicado el 31/01/2022

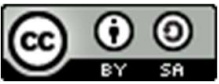

\section{PALABRAS CLAVE}

Tecnología de la comunicación, Psicología positiva, Comunicación personal, Educación a distancia.

\section{KEYWORDS}

Communication technology, Positive psychology, Personal communication, Distance education.

\section{RESUMEN}

El uso de las tecnologías educativas en la actualidad viene influenciando de manera constante en la enseñanza y aprendizaje, pues poseen múltiples herramientas digitales que favorecen el desarrollo intrapersonal e interpersonal de la inteligencia emocional. Por ello, la presente investigación tuvo por finalidad analizar la influencia de las herramientas digitales en la inteligencia emocional de los niños de inicial durante las clases virtuales en una escuela de Comas (Lima-Perú). Este objetivo orientó el estudio mediante el enfoque cualitativo de tipo estudio de caso, donde se aplicó una entrevista semiestructurada a docentes con experiencia en la enseñanza de niños de preescolar. Al concluir se obtuvo como resultados que las herramientas digitales sí influyen en la inteligencia intrapersonal fortaleciendo el autoconocimiento y la exploración de sus emociones. También, en la inteligencia interpersonal las herramientas digitales generan empatía, amigabilidad y liderazgo social. Y, por último, son importantes en la práctica del docente.

\section{ABSTRACT}

The use of educational technologies is currently influencing teaching and learning in a constant way, since they have multiple digital tools that favor the intrapersonal and interpersonal development of emotional intelligence. Therefore, the purpose of this research was to analyze the influence of digital tools on the emotional intelligence of preschool children during virtual classes in a school in Comas (Lima-Peru). This objective guided the study through a qualitative case study approach, where a semi-structured interview was applied to teachers with experience in teaching preschool children. At the conclusion, the results showed that digital tools do influence intrapersonal intelligence, strengthening self-knowledge and the exploration of emotions. Also, in interpersonal intelligence, digital tools generate empathy, friendliness and social leadership. And finally, they are important in the teacher's practice. 


\section{INTRODUCCIÓN}

Las Tecnologías de la Información y Comunicación (TIC) en la actualidad están logrando beneficiar y satisfacer las necesidades de las personas produciendo un avance tecnológico a nivel global (Kachakova, 2020). Por ejemplo, en los dos últimos años se ha registrado un crecimiento tecnológico en el contexto peruano, donde por cada 100 hogares 95 cuentan por lo menos con una tecnología (Instituto Nacional de Estadística e Informática [INEI], 2020). Asimismo, las tecnologías generan múltiples cambios en la sociedad, en el campo educativo se ha demostrado nuevos horizontes en la enseñanza y aprendizaje por medio de herramientas digitales, donde los estudiantes se sienten motivados y atraídos por aprender (Adarkwah, 2021). Este cambio educativo constituye ser la apertura de un nuevo paradigma tecnológico, que en países con un mejor desarrollo vienen fortaleciendo las prácticas educativas (Hatch, 2021).

Por consiguiente, las herramientas digitales en la enseñanza vienen beneficiando múltiples campos de la educación, en especial el desarrollo de la inteligencia emocional, la cual es definida como la capacidad de controlar y gestionar las emociones propias y ajenas, pues, uno de los desafíos de la escuela es lograr que los estudiantes autorregulen sus emociones y aprendan a socializar (Morales, 2020). Por otra parte, existen opiniones que señalan que vincular la tecnología con la educación no es una buena idea (Zilka, 2017), pero, para la educación inicial este cambio resulta ser una oportunidad favorable para enseñar, donde los docentes deben utilizar diversas estrategias que permitan a los niños un adecuado dominio de las emociones y de ese modo establecer una mejor relación con los demás (Masoumi, 2021). De esta manera, las herramientas digitales cumplen una función importante en la enseñanza de los alumnos, quienes ven en la tecnología la oportunidad de aprender de manera significativa (Mucundanyi y Woodley, 2021).

Ahora, en lo que respecta a la inteligencia emocional se consideran cinco dimensiones tales como: el autoconocimiento, la autorregulación, la motivación, la empatía y las habilidades sociales (Anand, 2019), las cuales se encuentran inmersas en el desarrollo intrapersonal e interpersonal de las personas (Sebastián y Garrido, 2017). De esta manera, se conoce que cada personalidad es distinta, pues los estados de ánimo están en constante cambio encontrando emociones de cólera, tristeza, miedo, inseguridad y desconfianza lo que puede perturbar la personalidad del infante (Sarmento-Henrique et al., 2020). Por ello, los docentes deben saber identificar como los niños expresan sus emociones para de ese modo plantear estrategias que ayuden a mejorar la inteligencia emocional (Hyoyeon et al., 2019; Masoumi, 2021). De esta manera, se ha demostrado que las herramientas virtuales a través del aula virtual son muy útiles en el aprendizaje emocional (Kamei y Harriott, 2021). Por ejemplo, para ayudar a los niños a familiarizarse y regular sus emociones, se pueden utilizar actividades que incorporen herramientas digitales como la narración digital, con sonidos ambientales, videos animados, música de fondo y proyecciones de la vida de los estudiantes (Mitsch et al., 2021), que, a través de dispositivos electrónicos como teléfonos celulares, cámaras, tabletas o 
computadoras, pueden ayudar a los niños a darse cuenta de su importancia, de dónde vienen, sus valores y su fuerza interior, controlando así mejor sus emociones (Rahiem, 2021).

Por otro lado, las herramientas digitales motivan a los alumnos en mejorar sus desempeños escolares, pues, plenamente demuestran muchas habilidades informáticas (Prensky, 2013). Por tanto, al realizar alguna actividad que vincule las tecnologías fortalecen sus aspiraciones, se sienten motivados (Sekhri, 2021) y facilitan la práctica docente (Konca y Koksalan, 2017). Durante las clases virtuales los docentes han adquirido nuevas maneras de proceder con la enseñanza, siendo el rol de facilitador que ahora define su trabajo, porque debido al uso de las herramientas digitales el estudiante no necesita del acompañamiento del docente de manera constante (Rodríguez, 2020). Asimismo, su empatía por comprender las situaciones difíciles en las que viven las personas se ha sensibilizado por medio de las actividades digitales en la enseñanza (Palmer, 2018; Bruno y Chelsea, 2018). También, como parte de la inteligencia emocional las habilidades sociales en la edad preescolar es un elemento importante que se tiene que desarrollar (Flecha et al., 2020), por tanto, mediante las herramientas tecnológicas se está logrando que los estudiantes aprendan a trabajar en grupo a través de interacciones virtuales (Blau et al., 2020; Gunawan et al., 2021), para así controlar sus emociones cuando se encuentran en conflicto, aprendan a respetar las emociones de sus compañeros y a la vez puedan interactuar entre ellos (Sánchez-Pacheco, 2020). De esta manera, los recursos digitales pueden ser empleadas en múltiples actividades (Blau et al., 2020), donde los docentes primeramente deben conocer y saber aplicar cada herramienta digital, para que la enseñanza y aprendizaje tenga mayor relevancia (Masoumi, 2021).

Finalmente, los recursos de la tecnología en el rol pedagógico deben entenderse como un nuevo modelo educativo, en que las escuelas deben utilizar nuevos recursos metodológicos para promover el aprendizaje (Eka, 2021; Hatch, 2021). De esta manera, la enseñanza virtual no necesariamente tiene que remplazar a las clases presenciales, sino que ambas pueden trabajar en conjunto para lograr el propósito de mejorar las competencias y capacidades de los estudiantes (Adarkwah, 2021). Es por ello que la importancia de las herramientas digitales en el rol docente se observa como una función virtual facilitadora, pues el estudiante construye su propio aprendizaje por medio de las indicaciones que el profesor le otorgue (Mohammed y Kinyo, 2020). Por consiguiente, se ha considerado el siguiente objetivo general: analizar la influencia de las herramientas digitales en la inteligencia emocional de los niños de inicial durante las clases virtuales en una escuela de Comas. Y, de manera específica identificar la influencia de las herramientas digitales en la inteligencia emocional intrapersonal de los niños de inicial durante las clases virtuales en una escuela de Comas. También, identificar la influencia de las herramientas digitales en la inteligencia emocional interpersonal de los niños de inicial durante las clases virtuales en una escuela de Comas. Y, finalmente analizar la importancia de las herramientas digitales en la inteligencia emocional de los niños de inicial en el rol pedagógico docente en una escuela de Comas. 


\section{MATERIALES Y MÉTODOS}

El enfoque de investigación corresponde al cualitativo de tipo descriptivo, cuya función permite la comprensión y descripción directa del objeto de estudio (Burgos et al., 2019). También, se empleará un estudio de caso el cual servirá para analizar los diversos aspectos del fenómeno de estudio (Ellinger y McWhorter, 2016).

\section{Participantes}

La muestra está constituida por dos docentes de la educación inicial de una escuela pública de comas, Lima, Perú. El tipo de muestreo corresponde a la selección no probabilística por conveniencia de acuerdo al interés del estudio (Taherdoost, 2016). La primera docente es Licencia de educación inicial con 35 años de experiencia, con especialización en las estrategias didácticas de enseñanza de Ciclo II y III en las áreas de matemática y comunicación y la segunda docente es Licencia de educación inicial con 15 años de experiencia.

\section{RESULTADOS Y DISCUSIÓN}

En la presentación del análisis de discusión y resultados se analizará los datos a través de un proceso de codificación y triangulación (Díaz, 2009). Primero, se analizará la influencia de las herramientas digitales en la inteligencia emocional intrapersonal. Segundo, la influencia de las herramientas digitales en la inteligencia emocional interpersonal. Y, por último, la importancia de las herramientas digitales en la inteligencia emocional de los niños de inicial en el rol pedagógico.

Influencia de las herramientas digitales en la inteligencia emocional intrapersonal

La inteligencia emocional es aquella que permite la capacidad de controlar y gestionar las emociones internas y externas (Morales, 2020). De manera que, sentir cólera, tristeza, miedo, inseguridad y desconfianza son algunas de las emociones intrapersonales que se deben aprender a superar (Sarmento-Henrique et al., 2020). Sin embargo, conseguir que todos logren un desarrollo emocional adecuado, no es una tarea fácil, por esta razón, surgen modos de desarrollar la inteligencia emocional a través de las herramientas digitales, como se puede evidenciar en las siguientes categorías emergentes:

\section{Los niños tienen más contacto con los aparatos electrónicos en especial los celulares}

Con el auge de la tecnología en la actualidad, las escuelas han implementado nuevas estrategias para la enseñanza y aprendizaje por medio de recursos digitales y tecnológicos, los cuales parecen captar mejor la atención de los educandos motivando su aprendizaje (Adarkwah, 2021), especialmente reciben esta atención los dispositivos móviles como los celulares por ser de fácil adquisición y manipulación. De manera que, una de las docentes entrevistadas mencionó su 
experiencia trabajando desde la modalidad remota, tal como se evidencia en el siguiente testimonio.

Sí, porque ahora con esta nueva situación desde el año pasado se estaba trabajando a distancia, los niños tienen más contacto con los aparatos electrónicos ya sea la laptop o celulares (...) manejan inclusive mejor estas herramientas, porque (...), ahora ya es una necesidad (...) más el celular, porque en esta situación los niños con los cuales yo trabajo la mayoría tienen celular y no cuentan con laptop (...) (DI-1).

Mediante el testimonio afirmado se puede evidenciar que los niños tienen más contacto, control y dominio con la tecnología (Prensky, 2013). En especial con los celulares, lo que demuestra que su implementación formaría parte de las estrategias de enseñanza pudiendo beneficiar el desarrollo de la inteligencia emocional de diferentes maneras, como se puede evidenciar en la siguiente categoría emergente.

\section{Medios para trabajar las emociones a través de herramientas digitales}

Existen múltiples formas para ayudar a los niños a familiarizarse y regular sus emociones por medio de las herramientas digitales (Rahiem, 2021). Respecto a ello una de las docentes entrevistadas manifestó el siguiente comentario.

Las herramientas digitales como su nombre lo dicen, nos va a permitir a nosotros poder utilizarlas y llegar de diferentes maneras (...) A través de una pequeña cámara y por ello que, de manera amigable tenemos que valernos de estas herramientas digitales para que, a través de diferentes imágenes, emoticones, mensajes, canciones, cuentos, juegos esencialmente podamos llegar a poder trabajar este tema del control de emoción gracias a las herramientas digitales (DI-2).

De esta manera, se puede apreciar que el uso de las herramientas digitales facilita la enseñanza (Hatch, 2021), empleando imágenes, emoticones, mensajes, canciones, cuentos y juegos por medio de las herramientas digitales pueden influir en las emociones intrapersonales de los infantes. Por tanto, cada docente debe valerse de ellas para ayudar a los niños a conocerse a sí mismos y fortalecer sus emociones internas. A continuación, se anunciará la siguiente categoría emergente.

\section{Las herramientas digitales para la exploración del niño}

Por otra parte, el uso del internet y los recursos tecnológicos tales como los celulares, Tablet u otros dispositivos han permitido que las personas lo usen como un medio de exploración, ya que otorgan múltiples beneficios personales y satisfacen las necesidades de los usuarios (Kachakova, 2020). Al respecto, una de las docentes entrevistadas afirmó el siguiente testimonio.

(...) Estas herramientas en este caso el internet (...) el celular que es el medio, la laptop que es medio de buscar la información a través de la internet, se puede decir que las herramientas digitales han servido como una herramienta de exploración hacia el niño (...) (DI-1).

Se puede afirmar que las herramientas tecnológicas durante las clases virtuales han sido de utilidad para la exploración del niño, donde no solo podrá encontrar 
contenido educativo, sino que también podrá distinguir y valorar todo tipo de información (Mucundanyi y Woodley, 2021), sirviendo como entrenamiento para el autoconocimiento de sus emociones intrapersonales, tal como se reflexionará en la siguiente categoría emergente.

\section{El autoconocimiento mediante una sesión de aprendizaje}

En esta categoría el autoconocimiento está orientado por medio del uso de las herramientas digitales como una estrategia (Kamei y Harriott, 2021), donde mediante una sesión de aprendizaje la maestra busca descubrir las emociones internas de los niños a través de los saberes previos, tal como lo señala una de las docentes entrevistadas.

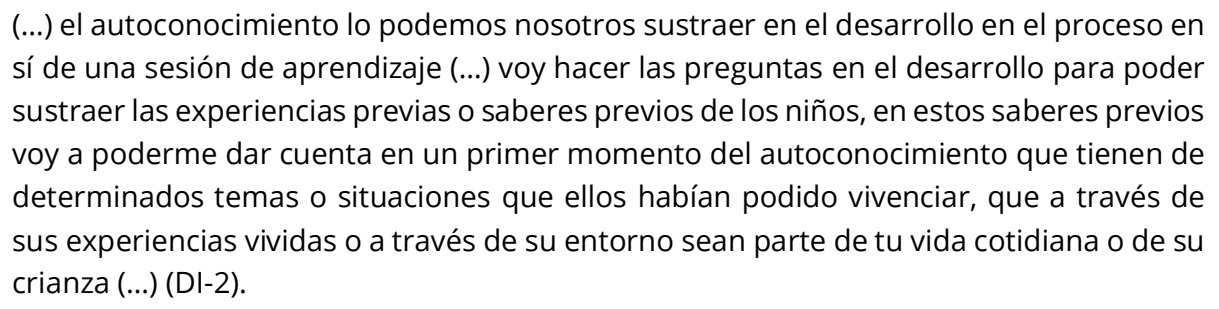

Un aspecto fundamental que señala la maestra es la productividad que tienen las herramientas digitales en el logro del autoconocimiento (Kamei y Harriott, 2021), detalle que para algunos docentes no es tan factible realizar, tal como se evidencia en la siguiente categoría emergente.

\section{Las TIC no ayudan en cierto sentido a las emociones}

El trabajo de las emociones por medio de las tecnologías si bien es cierto es un campo nuevo e interesante para algunos docentes, para otros viene generando dudas y descontentos, en especial cuando se le vincula con la inteligencia emocional (Masoumi, 2021), donde no todos afirman que sea un medio completamente efectivo, tal como lo señala una de las entrevistadas en el siguiente testimonio.

(...) ellos simplemente expresan sus emociones según como ellos lo sienten, en el momento, las TIC como se les dice yo creo que no los ayuda hasta cierto sentido, porque, ellos cuando quieren algo lo expresan hablando o lo expresan de otra manera con su cuerpo (...), porque los niños no entienden por decir que es la emoción, ellos te dicen porque estoy alegre, porque estoy triste, porque soy feliz, porque estoy molesto, entonces con el uso de las TIC a veces los niños ya no te expresan, no te lo dicen (...) (DI1).

Además, respecto a los emoticones como símbolos de las emociones la entrevistada afirmó.

(...) del uso de las caritas y todo eso ya a veces ellos los expresan, ahora el uso de estas caritas felices, triste y con la infinidad de caritas que hay ellos lo asocian a eso, a veces ya está perdiendo lo que es la comunicación (DI-1). 
Sin embargo, otras opiniones señalan que las herramientas digitales otorgan una interacción adecuada entre docente y estudiantes (Morales, 2020), tal como se reflexionará en la siguiente categoría emergente.

\section{El Zoom permite una interacción afectiva}

Respecto al Zoom se conoce que es una plataforma online donde se puede interactuar mediante sesiones de videoconferencias durante clases virtuales (Eka, 2021). Al respecto una de las docentes entrevistada manifestó su forma de trabajo mediante el Zoom.

(...) yo trabajo con Zoom me permite a mi poder estar a través de estos dos grupos (...) al mismo tiempo voy viendo esa interacción, esa confianza que existe entre nosotros, ya conoces que soy tu profesora cachetoncita, que abro los ojos, que me impresionó que me río y ahora vamos a bailar juntos, entonces esa confianza me va dando a mí la oportunidad de que comience a trazarte este lazo de afectividad entre nosotros ya que nos vemos todos los días (DI-2).

Se puede observar que para la maestra el trabajo por medio del aplicativo Zoom le permite una mejor interacción basada en la afectividad y confianza (Gunawan et al., 2021). Asimismo, existen otras maneras de trabajar las emociones con las herramientas digítales, las cuales generan interés y motivación a los infantes, tal como se evidencia en la siguiente categoría emergente.

\section{Aplicaciones que generan interés y motivación}

Por otra parte, por medio de las herramientas digitales existen aplicaciones que están influyendo sobre los intereses de los estudiantes (Kamei y Harriott, 2021), los cuales para las maestras entrevistadas son el WhatsApp, Zoom, YouTube, vídeo selfis y herramientas de se aprende en casa, tal como lo señalan las docentes entrevistadas en los siguientes comentarios.

Los niños ya se han adaptado al aprendizaje a través de este medio, entonces lo que estamos trabajando ahorita son el WhatsApp y el Zoom y video llamadas de WhatsApp (...) se podría decir una interacción más directa en comparación al año pasado, ellos te ven, ellos te escuchan, tú los observan que están haciendo, están trabajando con quienes están, de esta manera estamos interactuando con ellos (DI-1).

Además, la siguiente entrevistada planteó:

Bueno, por ejemplo, a mí me parece muy bueno el YouTube, lo trabajo al inicio con una música con una canción de dibujitos animados y la canción en la cual estén muchachos niños y que sean canciones de movimiento (...) con mi selfi estoy que me grabó mientras que hago mi compra (...) luego yo también para poder motivarlos según el tema, porque estamos trabajando con estrategia aprendo en casa (DI-2).

De esta manera, se puede observar que la influencia de las herramientas digitales primeramente genera motivación e interés en los estudiantes (Sekhri, 2021), siendo una alternativa para que los docentes pueden trabajar la inteligencia emocional. A continuación, se reflexionará la siguiente categoría general. 
Influencia de las herramientas digitales en la inteligencia emocional interpersonal

La inteligencia interpersonal son aquellas capacidades que una persona tiene para poder relacionarse por medio de habilidades sociales con los demás (Anand, 2019), teniendo en cuento esto, las maestras entrevistadas hicieron referencia a las siguientes categorías emergentes.

\section{Los niños tienen empatía por los estudiantes que no asisten a clase}

La empatía se manifiesta cuando una persona percibe las emociones y sentimientos de otros y se pone en su lugar (Bruno y Chelsea, 2018), tal como una de las maestras lo señala en su experiencia durante las clases virtuales.

(...) cómo me dijo una vez una niña: ah no ha hecho la recarga su mamá, mis que esta niña puede venir a mi casa me dice. Porque tengo niños que viven cerca, entonces comparten el internet, entran dos a la clase de zoom a través de un mismo celular (DI$1)$.

De esta manera, se observa que los niños suelen agruparse para poder realizar sus clases online cuando tienen problemas de internet, lo que señala que tanto en casa como en la escuela los niños desarrollan habilidades sociales (Flecha et al., 2020), tal como se evidencia en la siguiente categoría emergente.

\section{La maestra muestra un ambiente de amigabilidad y de confianza}

La escuela no solo debe ser entendida como un paraje de impartir conocimientos, sino también debe promover habilidades sociales que permitan al estudiante tener un mejor desarrollo de su personalidad (Masoumi, 2021), por tanto, los docentes en todo momento deben mostrar confianza y amigabilidad, tal como lo señala una de las maestras entrevistadas.

(...) yo más aspiro en que en todo ambiente, en todo ámbito escolar en el que estoy ya sea de niños o de grandes, que haya un ambiente de amigabilidad de confianza (DI-2).

La maestra al mostrar amistad y confianza ante los niños, está logrando que los alumnos reconozcan el valor de la amistad, por tanto, los docentes deben ser personas empáticas, tal como se evidencia en la siguiente categoría emergente.

\section{La empatía de la maestra durante las clases virtuales}

Utilizar la empatía como habilidad social permitirá al docente ganarse la confianza de sus estudiantes (Palmer, 2018), ya que ellos al sentirse escuchados y atendidos podrán superar los obstáculos, tal como lo señala una de las maestras entrevistadas.

Por ejemplo, la vez pasada tuve uno, en realidad varios que en clase se ponen a llorar, porque no pueden lograr algo (...) ya tenía conocimiento del niñito que se frustra y llora cuando no puede hacer algo, entonces ya empezó a llorar y yo delante de todos le saludo, hola Jordán qué pasa a ver cuéntame qué sucede, entre sollozos me dijo que no podía y le dije: pero no te preocupes, que ya lo vas a lograr poco a poco, tu mamita te va 
ayudar y poco a poco lo vamos a lograr, así de esa forma compartir con los otros niños (...) (DI-1).

La comunicación es una estrategia clave para poder solucionar las dificultades (Hyoyeon et al., 2019), pero también se puede usar otros recursos, tal como se evidencia en la siguiente categoría emergente.

\title{
4. Videos animados para lograr solucionar problemas emocionales
}

Por otra parte, el uso de los vídeos animados está teniendo cada vez más utilización por parte de las docentes de preescolar, porque seleccionan contenidos que estimulan la inteligencia emocional de los niños y que además pueden solucionar problemas (Mitsch et al., 2021), tal como una maestra afirma en el siguiente comentario.

\begin{abstract}
Bueno la experiencia me dice que cuando tienes a un niño que identificas que pasa por un problema emocional nunca vas a ser directa Ok. El tema se aborda de manera muy sutil (...) yo directamente lo que haría es trabajar a través de la búsqueda, eso lo tengo que hacer en un horario aparte la búsqueda de algún vídeo que vaya relacionado con el tema que él tiene entonces vamos a darnos cuenta temas muy interesante, te encuentras muchas cosas en YouTube en cuenta muchas cosas y no solamente en YouTube en otras páginas también en los cuales podemos ver ahí sí en dibujos porque el dibujo es lo que atrae al niño (...) (DI-2).
\end{abstract}

Es importante recalcar que a los niños les atrae los dibujos animados, siendo una buena estrategia que las maestras pueden utilizar para sensibilizar las emociones e influir en las habilidades sociales (Rahiem, 2021), tal como se evidencia en la siguiente categoría emergente.

\section{Las herramientas digitales ayudan en la socialización}

A pesar de que las herramientas digitales ya venían funcionando, muchos docentes desconocían que el uso tecnológico se podría emplear para enseñar a socializar (Sánchez-Pacheco, 2020), por tanto, una de las maestras manifestó lo valioso que ahora es para los niños.

Si influye porque ahora en esta situación en los niños se han quedado (...) entonces es muy importante en la socialización, como todo el tiempo es el uso de la tecnología, celular, la laptop, y la Tablet (DI-1).

De manera que, los celulares, Tablet y laptop en la educación virtual tienen una función crucial y estos pueden ser usados para enseñar a socializar a los estudiantes de manera interactiva (Kamei y Harriott, 2021), aunque estos presenten algunas limitaciones, como se puede evidenciar en la siguiente categoría emergente.

\section{Definitivamente las herramientas digitales te ayudan a socializar con limitaciones}

Si bien es cierto el uso de las herramientas digitales son de mucha ayuda para la enseñanza, no siempre cumplirá una función tan exacta por lo que presentará 
algunas limitaciones, pero sin embargo su existencia es necesaria para la comunicación (Adarkwah, 2021), como lo señala una de las maestras entrevistadas.

Definitivamente las herramientas digitales te ayudan a socializar con limitaciones (...) si no existieran estás herramientas digitales, cómo podrías obtener este apoyo este soporte este continuar tu aprendizaje, cómo podrías aprender a convivir con otros si solamente te has quedado de manera sedentaria con la comunicación únicamente de los miembros de tu familia, entonces sí contribuye efectivamente es un gran conocimiento del ser humano que tengamos estos avances en la tecnología (DI-2).

De acuerdo con lo mencionado, se puede observar que los tiempos han cambiado y que ahora mediante la nueva era digital los maestros tienen nuevas oportunidades de comunicarse, enseñar y socializar (Hatch, 2021), a pesar de que para otras personas se ha visto como algo negativo, como se evidencia en la siguiente categoría emergente.

\section{La influencia de las herramientas digitales para otras personas es contemplada de forma negativa}

La parte negativa de las herramientas tecnológicas radica en la libertad que el niño puede tener por medio del internet, es decir el contenido puede ser no apto para su edad lo que le puede generar conflictos emocionales (Zilka, 2017), por tanto, se aconseja que los niños tengan una debida orientación por sus padres a la hora de usar la tecnología, como se evidencia en el siguiente comentario de una de las maestras.

Muchas personas lo ven de lado negativo ya que se ponen a hacer comentarios que no son, pues no debería, pero como son niños pequeños, ellos no están accesos a las redes, ya eso depende mucho de los papás (...) pero cuando ya van creciendo estén de más edad, sí creo, pero por ahora son de inicial, todavía no lo entienden (DI-1).

Sin embargo, la idea de que muchas páginas de internet pueden ser dañinas para los niños no deben ser descuidadas por la orientación de la familia (Zilka, 2017), ya que en la escuela estas herramientas digitales se utilizan para facilitar el liderazgo social, como se evidencia en la siguiente categoría emergente.

\section{Las herramientas digitales facilitan el liderazgo social}

Las actividades educativas digitales a diferencia de la anterior categoría buscan generar y potenciar las capacidades de los estudiantes, siendo en este caso un medio ideal para el liderazgo social (Blau et al., 2020), tal como se comenta una de las docentes entrevistadas.

Bueno en este aspecto te puedo decir que, si influyen, pero para temas como en personal social voy a poder expresar mis ideas voy a poder participar voy a poder interactuar con amigos Ok. Bien el liderazgo se ve en el desarrollo de las actividades, los niños te dan propuestas, definitivamente las herramientas sociales nos van a facilitar (...) (DI-2).

De esta manera, se puede notar que en campo educativo las herramientas digitales pueden ayudar a los niños a desarrollar el liderazgo, por tanto, los docentes deben 
adecuarlas a la enseñanza y a las actividades escolares. Finalmente, se reflexionará en la última categoría general.

\section{Importancia de las herramientas digitales en el rol pedagógico}

Por otra parte, las herramientas digitales en la función pedagógica ya venían registrándose con anterioridad en la enseñanza, sin embargo, su uso no tenía mucha importancia como en la actualidad (Kachakova, 2020), teniendo en cuento esto, las maestras entrevistadas hicieron referencia a las siguientes categorías emergentes.

\section{Las herramientas son el brazo derecho de las maestras}

A pesar de que no todos los docentes antes de las clases virtuales valoren la importancia de las herramientas tecnológicas, en la actualidad estos medios se han convertido en un aleado estratégico para el rol pedagógico (Konca y Koksalan, 2017), llegando incluso a ser considerado como la mano derecha del docente, como se evidencia en el comentario de una de las maestras.

Bueno definitivamente ahora las herramientas digitales son como el brazo derecho de una maestra ya que a través de estas herramientas digitales me permiten llegar a los estudiantes me permiten motivar a los estudiantes me permiten desarrollar competencias en los estudiantes me permiten tener ese contacto y comunicación no solamente con ellos sino por los padres de familia quiénes van a ser mi otro brazo también para poder garantizar que lo que estamos trabajando se pueda desarrollar en casa (DI2).

Esta nueva perspectiva de la tecnología en la educación está permitiendo que los estudiantes tengan mayor motivación, pues desarrollan competencias digitales con mayor interés y rapidez (Sekhri, 2021), como se puede evidenciar en la siguiente categoría emergente.

\section{Los niños son más tecnológicos}

Los cambios que la educación virtual han traído a la pedagogía son considerados por los docentes como un nuevo enfoque que revaloriza la forma de aprender y de enseñar (Hatch, 2021), situación que ha generado en los estudiantes una mayor interacción que antes no había en el sistema educativo, tal como lo menciona una de las maestras en el siguiente comentario.

(...) ahora los niños son como tú lo dijiste, más tecnológicos y ellos a veces a través de esta situación se han vuelto más extrovertidos, lo que no era en un principio, ahora ya lo es a través de la pantalla a pantalla, no sé cómo será cuando esté presencial los niños tendrán la misma actuación no sé, pero a través de la situación si yo lo he visto porque desde marzo habido un cambio, de niños calladitos que eran, ahora sí te hablan, se conversan, se mueven, corren, saltan y gritan (DI-1).

De esta manera, se puede notar un cambio en los estudiantes de la educación inicial, quienes a pesar de tener una corta edad saben usar muy bien los medios tecnológicos (Konca y Koksalan, 2017; Prensky, 2013), lo que lleva a los docentes a 
cumplir una función más facilitadora del aprendizaje, como se puede evidenciar en la última categoría emergente.

\section{El docente como facilitador en la enseñanza virtual}

Durante las clases virtuales los docentes han adquirido nuevas maneras de proceder con la enseñanza, siendo el rol de facilitador que ahora define su trabajo, porque debido al uso de las herramientas digitales el estudiante no necesita del acompañamiento del docente de manera constante (Rodríguez, 2020), de acuerdo a ello una de las maestras entrevistadas hizo el siguiente comentario.

Pero seguimos siendo facilitadores. Porque esa oportunidad no tanto le damos los materiales en concreto para que los niños lo manipulen directamente, pero si le damos para facilitar materiales de tipo virtual a través de los diferentes programas como te decía vamos a hacer el rol de orientadores, de guías solamente para el niño, sino también vamos a tener que ser las guías de las madres de los padres o de esa abuelita o tía que está al lado para que ella pueda desarrollar las actividades (...) (DI-2).

Finalmente, en la importancia de las herramientas digitales en el rol del docente se puede observar que su función virtual es solo de facilitador, pues el estudiante construirá su propio aprendizaje por medio de las indicaciones que el profesor le otorgue (Mohammed y Kinyo, 2020).

\section{CONCLUSIONES}

En conclusión, el uso de las herramientas digitales viene influyendo y desempeñado un papel importante para la enseñanza general y de la inteligencia emocional dentro del campo intrapersonal e interpersonal de los infantes, pues estas motivan y generan el interés de los estudiantes, como también facilitan la enseñanza de los docentes. Y, de manera específica se concluye que las herramientas digitales sí influyen dentro de la inteligencia intrapersonal, pues los niños tienen más contacto con los dispositivos electrónicos, como por ejemplo el celular, donde se pueden trabajar actividades con imágenes, emoticones, mensajes, canciones, cuentos y juegos por medio del Zoom y otras aplicaciones que generan motivación e interés, siendo ideal para el autoconocimiento y la exploración intrapersonal del niño. Asimismo, en la inteligencia interpersonal se concluye que las herramientas digitales a pesar de tener ciertas limitaciones si influyen en las emociones de los infantes, pues en el trabajo de la socialización se demostró la empatía, amigabilidad, liderazgo y confianza en las actitudes de los estudiantes y maestros. Finalmente, en la importancia de las herramientas digitales en el rol docente, se concluye que la nueva modalidad de enseñanza virtual se ha convertido en el brazo derecho de los educadores, quienes además asumen el papel de facilitadores; pues los niños han demostrado tener buenas competencias en el uso de las herramientas digitales.

En relación con lo expuesto, esta investigación tuvo como limitaciones el tamaño de la muestra y el análisis general de la inteligencia emocional. Por tanto, se sugiere para futuras investigaciones analizar las dimensiones de la inteligencia emocional de manera independiente a través de las siguientes temáticas: herramientas 
digitales para fortalecer el autoconocimiento, la autorregulación y su relación con las TIC, programas virtuales para trabajar la empatía y estrategias virtuales para promover motivación y habilidades sociales. Además, ampliar la muestra y profundizar el estudio por medio de una bibliografía más actualizada y de diversos contextos internacionales.

\section{AGRADECIMIENTOS}

Nuestro agradecimiento primero a Dios por que nos dio el don de la perseverancia para alcanzar nuestras metas. Así como también a las personas que de uno u otro modo colaboraron en la realización de esta investigación y a nuestras familias por su apoyo, comprensión y confianza.

\section{REFERENCIAS}

Adarkwah, M. (2021). I'm not against online teaching, but what about us: ICT in Ghana post Covid-19. Education and Information Technologies, 26(2), 16651685. https://doi.org/10.1007/S10639-020-10331-Z

Anand, P. (2019). Emotional Intelligence and Positive Education: Preparing Students for a Better Tomorrow. New Directions for Teaching and Learning, 2019(160), 107-116. https://doi.org/10.1002/TL.20368

Blau, I., Shamir, T., y Hadad, S. (2020). Digital collaborative learning in elementary and middle schools as a function of individualistic and collectivistic culture: The role of ICT coordinators' leadership experience, students' collaboration skills, and sustainability. Journal of Computer Assisted Learning, 36(5), 672-687. https://doi.org/10.1111/JCAL.12436

Burgos, P., Marquez, A., Baquerizo, B., Dolores, N., Alejandro, F., y Esmeralda, G. (2019). Métodos y técnicas en la investigación cualitativa. Algunas precisiones necesarias. Conrado, 15(70), 455-459.

http://scielo.sld.cu/scielo.php?script=sci_arttext\&pid=S199086442019000500455

Bruno, C., y Chelsea, D. (2018). Combining the Best Aspects of Humanity With the Best of Technology. Childhood Education, 94(6), 25-32. https://eric.ed.gov/?q=Technology+and+empathy\&id=EJ1195167

Díaz, M. (2009). ¿Cómo desarrollar, de una manera comprensiva, el análisis cualitativo de los datos? Educere, 13(44), 55-66. https://www.redalyc.org/pdf/356/35614571007.pdf

Eka, R. (2021). Application of the Zoom Meeting Application in Online Learning During the Pandemic. Education Quarterly Reviews, 4(2), 26-32. https://files.eric.ed.gov/fulltext/EJ1300621.pdf

Ellinger, A., y McWhorter, R. (2016). Qualitative Case Study Research as Empirical Inquiry. International Journal of Adult Vocational Education and Technology (IJAVET), $7(3)$, 1-13. https://eric.ed.gov/?q=what+is+the+case+study\&id=EJ1155655 
Flecha, R., Pulido, C., Villarejo, B., Racionero, S., Redondo, G., y Torras, E. (2020). Effects of the use of digital technology on children's empathy and attention capacity: analytical report. https://eric.ed.gov/?q=Technology+and+empathy\&id=ED607413

Gunawan, G., Kristiawan, M., Risdianto, E., y Monicha, R. (2021). Application of the Zoom Meeting Application in Online Learning during the Pandemic. Education $\begin{array}{lll}\text { Quarterly } \quad \text { Reviews, 26-32. } & \end{array}$ https://eric.ed.gov/?q=Zoom+to+interact\&id=EJ1300621

Hatch, B. (2021). A New Paradigm for Early Childhood Education: Opening doors through virtual kindergarten. Childhood Education, 97(2), 60-63. https://doi.org/10.1080/00094056.2021.1900768

Hyoyeon, I., Hyunhee, K., y Carney, J. (2019). The relation of social skills and school climate of diversity to children's life satisfaction: The mediating role of school connectedness. Psychology in the Schools, 56(6), 1023-1036. https://doi.org/10.1002/PITS.22247

Instituto Nacional de Estadística e Informática. (2020). Acceso de los hogares a las Tecnologías de Información y Comunicación (TIC). https://www.inei.gob.pe/media/MenuRecursivo/boletines/boletin_tics.pdf

Kachakova, V. (2020). The Potential of ICTs in Education Worldwide: A Review of Several International Studies. Bulgarian Comparative Education Society, 18(2), 77-81. https://eric.ed.gov/?q=ICT+impact\&ff1=dtySince_2020\&id=ED608403

Kamei, A., y Harriott, W. (2021). Social Emotional Learning in Virtual Settings: Intervention Strategies. International Electronic Journal of Elementary Education, 13(3), 365-371. https://files.eric.ed.gov/fulltext/EJ1297841.pdf

Konca, A., y Koksalan, B. (2017). Preschool Children's Interaction with ICT at Home. International Journal of Research in Education and Science, 3(2), 571-581. https://eric.ed.gov/?q=ICT+in+preschool+children\&id=EJ1148419

Masoumi, D. (2021). Situating ICT in early childhood teacher education. Education and Information Technologies, 26(3), 3009-3026. https://doi.org/10.1007/S10639-020-10399-7

Mitsch, M., Riggleman, S., y Buchter, J. (2021). Responding to Young Children's SocialEmotional Needs Through Video Modeling. Young Exceptional Children, 24(1), 16-27. https://doi.org/10.1177/1096250620910708

Mohammed, S., y Kinyo, L. (2020). Constructivist Theory as a Foundation for the Utilization of Digital Technology in the Lifelong Learning Process. Turkish Online Journal of Distance Education, 21(4), 90-109. https://eric.ed.gov/?q=digital+constructivism\&id=EJ1269609

Morales, M. (2020). Emotional intelligence and ethics of solidarity-based responsibility: how to contribute today to pedagogy. Revista de Ciencias de La Comunicación e Información, 25(2), 55-70.

http://revistaccinformacion.net/index.php/rcci/article/view/111/83

Mucundanyi, G., y Woodley, X. (2021). Exploring Free Digital Tools in Education, International Journal of Education and Development using Information and Communication Technology, 2021. Nternational Journal of Education and Development Using Information and Communication Technology, 17(2), 103- 
2021.

https://eric.ed.gov/?q=Digital+tools+for+selfawareness\&ff1=dtyln_2021\&id=EJ1297885

Palmer, J. (2018). Empathy. Brock Education: A Journal of Educational Research and Practice, 27(2), 34-37. https://eric.ed.gov/?q=empathy\&id=EJ1179925

Prensky, M. (2013). Enseñar a nativos digitales. Revista Electrónica de Investigación Educativa, 17(2), 1-3. https://www.redalyc.org/pdf/155/15537098002.pdf

Rahiem, M. (2021). Storytelling in early childhood education: Time to go digital. International Journal of Child Care and Education Policy, 15(1), 1-20. https://doi.org/10.1186/S40723-021-00081-X

Rodríguez, M. (2020). Rol del docente y estudiante en la educación virtual. Revista Multi-Ensayos, 6(12),

28-37. https://doi.org/10.5377/MULTIENSAYOS.V6I12.10117

Sánchez-Pacheco, C. (2020). Las redes sociales y las habilidades de enseñanza Aprendizaje del siglo XXI. Eduser (Lima), 7(1), 49-55. https://doi.org/10.18050/EDUSER.V7I1.2511

Sarmento-Henrique, R., Quintanilla, L., Lucas-Molina, B., Recio, M., y Giménez, D. (2020). The longitudinal interplay of emotion understanding, theory of mind, and language in the preschool years. International Journal of Behavioral Development, 44(3), 236-245. https://doi.org/10.1177/0165025419866907

Sebastián, E. y Garrido, M. (2017). Desarrollo de la inteligencia interpersonal e intrapersonal en educación primaria a partir del uso de tecnologías de información y comunicación: estudio de casos. Notandum 44-45 http://www.hottopos.com/notand44/14HerederoGarrido.pdf

Sekhri, A. (2021). Information and Communication Technology Emerges as a Beacon of Hope in Online Teaching. Shanlax International Journal of Education, 9(4), 294-299. https://doi.org/10.34293/education.v9i4.4161

Soriano, A. (2015). Diseño y validación de instrumentos de medición. Diá-Logos, (14), 19-40.

https://doi.org/10.5377/dialogos.v0i14.2202

Taherdoost, H. (2016). Sampling Methods in Research Methodology; How to Choose a Sampling Technique for Research. International Journal of Academic Research in Management (IJARM), 5, 5. https://hal.archives-ouvertes.fr/hal-02546796

Zilka, G. (2017). Awareness of eSafety and Potential Online Dangers among Children and Teenagers. Journal of Information Technology Education: Research, 16, 319-338.

https://eric.ed.gov/?q=the+danger+of+surfing+the+internet\&id=EJ1161792 